\title{
The Effect of Changes in Position of Sound Source and Microphone on Acoustic Measurements in Small Rooms. A Case Study
}

\author{
D. MleczKo* AND T. WSZOEEK \\ AGH University of Science and Technology, Department of Mechanics and Vibroacoustics \\ Al. A. Mickiewicza 30, 30-059 Krakow, Poland
}

\begin{abstract}
Results of computer simulations and measurements in real interior for varying location of sound source and microphone are shown in the paper. A small room with a volume of $47 \mathrm{~m}^{3}$ was used for this purpose. The objective of measurements and calculations was to determine the sound pressure level and other parameters derived from the room impulse response $\left(T_{30}, \mathrm{EDT}, C_{80}\right.$, STI) followed by the sensitivity analysis of those parameters to changing the location and orientation of the sound source and the receiver. In order to determine these parameters, the room impulse response was measured using MLS method. Experimental studies have been used to verify the acoustic room model built with use of enhanced radial method algorithms and its sensitivity. That allowed complementary and extended simulation studies on the room acoustic characteristics and finally determination of sensitivity of output parameters to changes of location and orientation of the measurement channel elements.
\end{abstract}

DOI: 10.12693/APhysPolA.125.A-131

PACS: 43.20.Dk, 43.50.Jh, 43.55.Ka, 43.58 Ta, 43.55.-n, 43.55.+p

\section{Introduction}

Computer programs are increasingly used in the room acoustics design work but the problem of determination of reliability of these simulations remains still unresolved. It is necessary to know many input values to build a room model and conduct a study. In many cases these values are variable and therefore their precise determination is difficult and sometimes even impossible. Therefore, it is useful to know to what extent inaccurate determination of some of these parameters may influence the final result of modeling.

It is necessary to determine both the acoustic measurement and simulation results uncertainties in order to determine the degree of divergence between simulation results and actual values.

The research presented in this paper, which is a part of a larger work [1], focuses on the uncertainty of acoustic measurements of the room.

Accordingly, the following factors are proposed to be included in the parameters influencing the prediction result:

- related to the sound source and the receiver:

- sound power and directivity of the sound source,

- measurement chain elements location (the guidelines for the study of interior acoustic

\footnotetext{
*corresponding author; e-mail: dmleczko@agh.edu.pl
}

space, defined in ISO 3382 [2] provide information related to the location of the measuring equipment items such as the sound source and the receiver, however the guidelines seem not to be accurate enough);

- related to the object:

- sound absorption coefficients of all surfaces in the modeled room,

- room model geometry accuracy,

- humidity, temperature, and air pressure;

- related to software:

- uncertainty introduced by the software used.

Uncertainties in acoustic prediction were studied only recently. In the paper [3] it was shown what accuracy is required when reporting the position of a receiver was used for room acoustic measurements ( $C_{80}$ was discussed). [4] deals with the influence of the source (dodecahedron speakers) on the measurement result. [5] reports that directionality of sound sources could cause wide audience areas to break away from this criterion at high frequencies, even when using dodecahedron loudspeakers which meet requirements of the ISO 3382 standard.

The effect of changes of some input parameters on the measurements and acoustic modeling of interiors was considered in some earlier publications [3-5], but none of them deal with all of possible parameters. Therefore the authors of the present paper made an attempt to determine input parameters that have the greatest effect on the final result of acoustic modeling of interiors and their accuracy is of particular importance. 
In the paper [6], the effect of environmental conditions on acoustic measurements was researched, while in the present paper, an attempt was made to determine how changing the measurement chain location (location and orientation of the sound source and orientation of the receiver) affects the result of measurements and calculations of reverberation time $T_{30}$, early decay time EDT, clarity $C_{80}$, speech transmission index STI, and sound power level SPL.

\section{Sample model}

Measurements were performed in a cube-shaped room with dimensions of about $6.7 \mathrm{~m} \times 4 \mathrm{~m}$ and height of $2.7 \mathrm{~m}$ (Fig. 1). The room was relatively small, its cubature was $72.88 \mathrm{~m}^{3}$, while the sum of area of all surfaces was $137.04 \mathrm{~m}^{2}$.

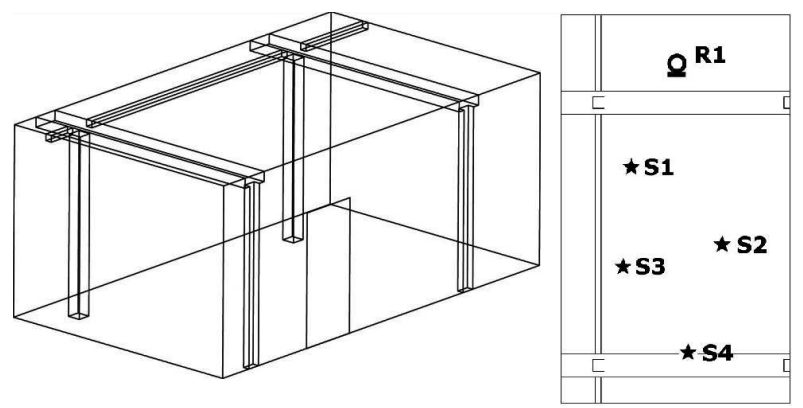

Fig. 1. Left: view of the room; right: location of the sound source (S1-S4) and measurement point (R1).

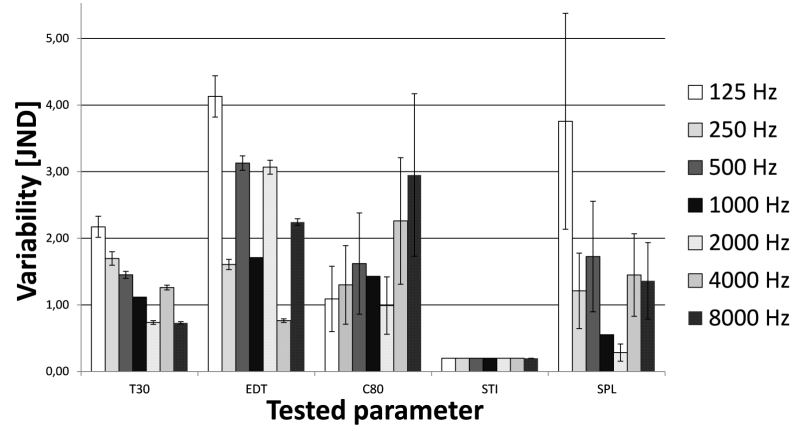

Fig. 2. Measurement results for variable position of the sound source in the room [1].

Measurements were performed using MLS method which served to determine the impulse response (Fig. 2). And on that basis, indicators to assess the quality of acoustic space were calculated. Specially constructed semi-spherical sound source (described in more detail in [7]) was used to study the interior acoustics. Measuring microphone PCB 377B20 ( $\frac{1}{2}^{\prime \prime}$ random incidence microphone), via the microphone amplifier (GRAS type 12AA) was connected to the input of a PC sound card (M-Audio Fast Track Ultra). DIRAC software recorded the room impulse response.
At the same time, the sound pressure level was measured. The measurements were carried out using SVANTEK 959 sound level meter. The sound pressure level values in octave bands and the A-weighted sound pressure level were recorded.

Experimental studies have determined $T_{30}$, EDT, $C_{80}$, STI and SPL and were designed to obtain answers to questions about:

- repeatability of measurement results - a series of 10 measurements with fixed parameters of the sound source and the microphone location;

- the effect of changes in orientation of the microphone with respect to the sound source - a study done by rotating the microphone around the tripod axis by $360^{\circ}$ in increments of $45^{\circ}$;

- the effect of changes in orientation of the sound source with respect to the microphone - sound column during the measurement was rotated around its axis by $360^{\circ}$ in increments of $60^{\circ}$. During the measurements, the microphone was facing the sound source;

- the effect of location of the sound source with respect to the microphone - measurements were made using four different randomly selected positions of the sound source. At the same time the microphone remained in fixed position.

In the latter two cases, only the measurement results were taken into account. When microphone and the sound source meet the condition of omnidirectionality (according to ISO 3382-1 [5]), they are usually modeled as perfectly omnidirectional.

Change of these input parameters affected the measured value of above mentioned output quantities. In order to enable comparison of these output quantities, their uniformity was introduced by using reference values according to ISO 3382-1 [2]. This ensures that all values can be expressed with the same unit, based on psycho-physical sensations, called just noticeable difference (JND). The reference values used in this study are presented in Table I (based on [2]). If uncertainties are smaller than this values, the simulation can be considered as sufficiently accurate (in accordance with the statement "don't compute what you can't hear" $[8,9])$.

\section{Results}

The repeatability of measurements was tested at the beginning of the experiment. It appeared that repeatability was good, except for $63 \mathrm{~Hz}$ octave band, where a large divergence of results was related to dimensions of the room and too low signal-to-noise ratio. Simultaneously it could be noted that only the results of $T_{30}(3.95$ JND) and EDT (2.57 JND) were affected by this problem. Therefore, further analysis did not take into account the $63 \mathrm{~Hz}$ octave band. In Figs. 3 and 4 and in Table II 


\section{TABLE I}

Average values of the perception threshold of the variation of specific parameters assumed in this paper as the reference values [2].

\begin{tabular}{l|c|c}
\hline \hline \multicolumn{2}{c|}{ Parameter (PN-EN ISO 3382-1) } & JND \\
\hline reverberation time & $T_{30}$ & $5 \%$ \\
early decay time & EDT & $5 \%$ \\
definition & $D_{50}$ & 0.05 \\
clarity & $C_{80}$ & $1 \mathrm{~dB}$ \\
sound strength & $\mathrm{G}$ & $1 \mathrm{~dB}$ \\
speech transmission index & $\mathrm{STI}$ & 0.05
\end{tabular}

the measurement repeatability results are expressed as the difference between the highest and the lowest value obtained from 10 measurements for each octave band.

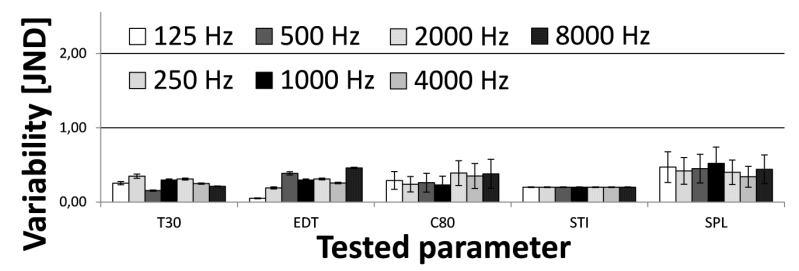

Fig. 3. Calculation results for variable position of the sound source in the room.

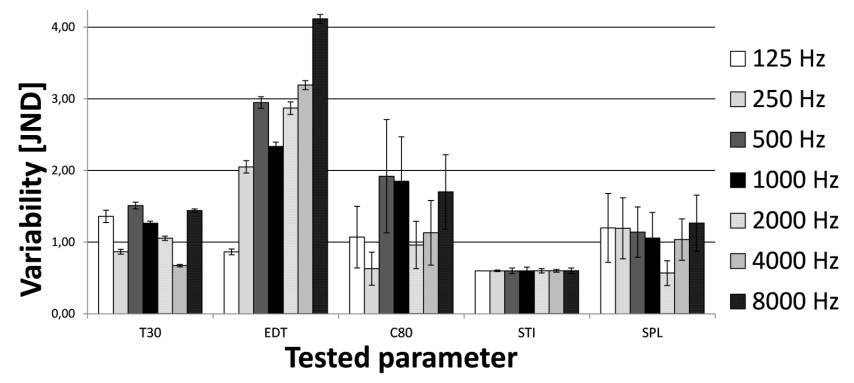

Fig. 4. Measurement results for variable orientation of the microphone in the room $\left(360^{\circ}\right.$ with a resolution of $\left.45^{\circ}\right)$.

Next, measurements and calculations were made to investigate the effect of changes in the sound source and the microphone location on the obtained results. Location of the sound source in the room has been modified at first. In that way, a series of four measurements was performed. Then the differences (within each octave band) between the smallest and the largest value obtained from all four measurements were calculated. The results together with standard deviation values are shown in Figure 2 (measurement) and Figure 3 (calculations). The procedure to determine the impact of changes in location of the microphone and the sound source was similar. The results are illustrated in Figure 4 (change of microphone orientation) and Figure 5 (change of sound source orientation).

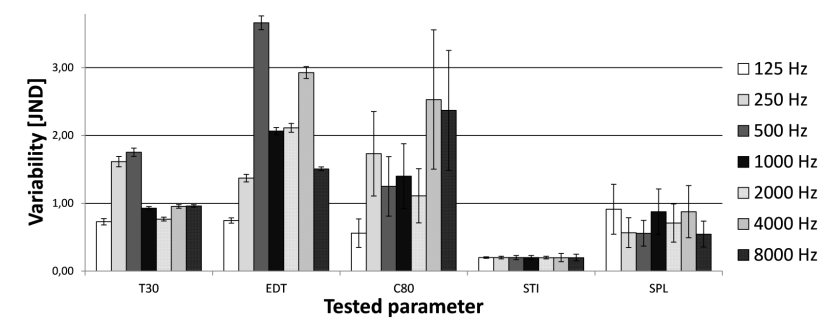

Fig. 5. Measurement results for variable orientation of the sound source in the room $\left(360^{\circ}\right.$ with a resolution of $60^{\circ}$ ).

TABLE II

Repeatability of measurement results. Results are the difference between the lowest and the highest values obtained.

\begin{tabular}{l|c|c|c|c|c|c|c|c}
\hline \hline & \multicolumn{7}{|c}{ Changes of the threshold of perception (in JND) } \\
\cline { 2 - 9 }$f[\mathrm{~Hz}]$ & 63 & 125 & 250 & 500 & 1000 & 2000 & 4000 & 8000 \\
\hline$T_{30}$ & 3.95 & 0.40 & 0.45 & 0.29 & 0.20 & 0.40 & 0.45 & 0.19 \\
EDT & 2.57 & 0.18 & 0.02 & 0.21 & 0.41 & 0.26 & 0.74 & 0.52 \\
$C_{80}$ & 0.15 & 0.03 & 0.02 & 0.05 & 0.08 & 0.22 & 0.19 & 0.18 \\
$D_{50}$ & 0.00 & 0.00 & 0.00 & 0.20 & 0.20 & 0.20 & 0.40 & 0.20 \\
STI & \multicolumn{7}{|c|}{0.00} \\
SPL & 0.43 & 0.04 & 0.05 & 0.02 & 0.07 & $0.10 \mid 0.17$ & 0.19
\end{tabular}

\section{Conclusions}

The research was performed to specifically determine the influence of changes in position of the sound source and the microphone on acoustic measurements in the room. On the grounds of the obtained results it can be concluded that:

- Accuracy of determination of the position of the sound source in the room, when placing it in a room with long reverberation time, at a similar distance from the receiver, is important when measuring $T_{30}$, EDT, $C_{80}$, and SPL (up to 4 JND for 125 $\mathrm{Hz}$ octave band). It can be expected that the test results will be more dependent on the location of the sound source in rooms with larger sound absorption.

- Orientation of the microphone with respect to the sound source has the greatest effect on measurement results of EDT (from 0.86 to 4.11 JND - the higher octave band center frequency, the greater possible divergence of the results), much less in case of $T_{30}$ (from 0.87 to $1.51 \mathrm{JND}$ ), and $C_{80}$ (from 0.63 to 1.92 JND). However, in case of STI (0.6 JND) and SPL (0.57 to 1.27 JND), orientation of the microphone had a negligible effect on the obtained results.

- As in the case of changes in orientation of the microphone, the change in orientation of the sound source has the greatest impact on the measurement 
of EDT (0.75 to 3.66 JND). To a lesser extent, the effect is noticeable in the measurements of $T_{30}(0.73$ to $1.75 \mathrm{JND})$ and $C_{80}(0.56$ to $2.53 \mathrm{JND})$. However, reorienting the sound source has only a negligible effect on measurements of STI (0.2 JND) and SPL (0.55 to $0.88 \mathrm{JND})$. It should be noted, however, that those values are strictly dependent on properties of the used source (its directional characteristics).

\section{References}

[1] D. Mleczko, Doctoral thesis, AGH University of Science and Technology, Kraków 2004.

[2] ISO 3382-1:2009, Acoustics - Measurement of room acoustic parameters - Part 1: Performance spaces.

[3] I. Witew, P. Dietrich, D. de Vries, M. Vorländer, in: Proc. Int. Symp. Room Acoustics (ISRA 2010), Australian Acoustical Society, Melbourne 2010.
[4] I. Witew, G. Behler, Forum Acust. 2005, Eds.: F. Augusztinovicz, A.B. Nagy, Z. Hunyadi, OPAKFI Tudomanyos Egyesület, Budapest 2005, p. 2291.

[5] R. San Martin, M. Arana, J. Acous. Soc. Am. 86, 133 (2008).

[6] D. Mleczko, P. Kleczkowski in: 59th Open Seminar of Acoustics, Ed. A. Józefczak, Polish Acoustical Society, Poznań Division, Poznań - Boszkowo 2012, p. 179.

[7] D. Mleczko, T. Wszołek, in: 35rd Winter School Vibroacoustical Hazards Suppression, Ed. M. Roczniak, Upper Silesian Division of the Polish Acoustical Society, Gliwice - Ustroń 2007, p. 111.

[8] M. Vorländer, in: Proc. Baltic-Nordic Acoustics Meeting (BNAM 2010), Bergen 2010, CD.

[9] M. Vorländer, in: International Seminar on Virtual Acoustics, Universitat Politècnica de València, València 2011 , p. 72 